\title{
Aplicação do Modelo de Aceitação de Tecnologia para uma Técnica de Inspeção de Usabilidade
}

\author{
Tayana Conte ${ }^{1}$, Verônica T. Vaz ${ }^{2}$, David Zanetti ${ }^{2}$, Gleison Santos ${ }^{3}$, \\ Ana Regina Rocha ${ }^{2}$, Guilherme H. Travassos ${ }^{2}$ \\ ${ }^{1}$ DCC - UFAM, CEP 69077-000, Manaus, AM, Brasil \\ ${ }^{2}$ PESC - COPPE/UFRJ, Cx Postal 68.511, CEP 21945-970, Rio de Janeiro, RJ, Brasil \\ ${ }^{3}$ UNIRIO, /DIA/CCET, Av. Pasteur, 458, CEP 22290-240, Rio de Janeiro, RJ, Brasil \\ tayana@dcc.ufam.edu.br, veronica.taquette@ufrj.br, gleison@uniriotec.br, \\ \{zanetti, darocha, ght\}@cos.ufrj.br,
}

\begin{abstract}
Users' acceptability of Web applications relies strictly on these applications' usability, which can be improved by carrying out inspections. This paper reports a case study of usability inspection using a technique called WDP. Our goal is to analyze the technique's effectiveness and appropriateness through the Technology Acceptance Model (TAM), in order to encourage its adoption by other software development companies.

Resumo. A aceitabilidade das aplicações Web por seus usuários depende diretamente da usabilidade da aplicação, que pode ser melhorada através da realização de inspeções. Este artigo relata um estudo de caso de inspeção de usabilidade utilizando uma técnica chamada WDP. O objetivo é analisar a eficiência e a adequação da técnica ao ambiente industrial através do Modelo de Aceitação de Tecnologia (TAM), de forma a incentivar a sua adoção em outras empresas de desenvolvimento de software.
\end{abstract}

\section{Introdução}

Usabilidade é um dos aspectos relacionados à qualidade em uso de sistemas, sendo um dos mais importantes critérios de aceitação para aplicações interativas em geral, e, em particular, para aplicações Web (Hitz et al., 2006). A importância da usabilidade é ainda maior em aplicações Web devido às suas características, pois "aplicações Web são aplicações interativas, centradas no usuário e baseadas em hipermídia, onde a interface com o usuário desempenha um papel central" (Olsina et al., 2006). Devido à relevância da usabilidade no contexto de aplicações Web, diversas técnicas e ferramentas específicas têm sido propostas com o intuito de melhorar tal atributo de qualidade. Apesar disso, muitas organizações de desenvolvimento Web não as estão aplicando (Insfran e Fernandez, 2008).

É necessário entender porque, embora a usabilidade seja um aspecto tão importante para as aplicações Web, tão poucas organizações de software Web utilizam avaliações de usabilidade durante seus processos de desenvolvimento. Uma questão específica é porque estas novas tecnologias desenvolvidas na academia não são transferidas para a indústria. Prováveis causas são: desconhecimento dessas técnicas; ou ainda o custo presumido de sua utilização. Uma importante causa que precisa ser discutida é a questão de quantas das novas tecnologias propostas são formuladas através de princípios científicos e transferidas de forma segura para a indústria.

Ao se decidir adotar uma nova tecnologia, é importante ter evidências disponíveis relativas aos benefícios, limitações, custos e riscos destas tecnologias, respondendo questões essenciais para a decisão de adoção de uma tecnologia (Travassos, 2008): (i) 
Quanto treinamento/investimento é necessário para inserir a tecnologia de software no meu processo? (ii) Sob que condições a tecnologia apresenta melhor desempenho? (iii) Quando e como eu poderei observar o Retorno de Investimento realizado?

Estudos experimentais são os "blocos de construção do conhecimento" necessários para construir evidências e determinar quais as melhores situações para empregar determinada tecnologia. Estudos in vivo são estudos que envolvem pessoas em seu próprio ambiente de trabalho em condições realistas (Travassos e Barros, 2003). Estudos de caso feitos em ambiente industrial são um tipo importante de estudo in vivo, visto que estes permitem a análise de um processo particular no contexto de um ciclo de vida de software (Shull et al., 2001). Este tipo de estudo é imprescindível para a indústria decidir se adota ou não uma nova tecnologia.

Neste sentido, este artigo relata um estudo de caso em uma organização de desenvolvimento de software, com uma técnica de inspeção de usabilidade específica para aplicações Web chamada WDP (Conte et al., 2009a). Neste estudo, desenvolvedores e avaliadores de requisitos utilizaram a técnica em questão para a avaliação de uma aplicação Web. O objetivo deste artigo é descrever como foi executada essa inspeção e analisar seu custo-benefício. O artigo também apresenta como a técnica foi avaliada em termos de adequação ao ambiente industrial através do modelo experimental TAM (Technology Acceptance Model) (Davis, 1989).

O restante do artigo está organizado da seguinte forma: a Seção 2 apresenta um referencial teórico sobre avaliações de usabilidade e a técnica WDP. A Seção 3 descreve a inspeção de usabilidade executada. A Seção 4 discute os resultados obtidos com a inspeção e a avaliação de adequação da técnica ao ambiente industrial. Por fim, a Seção 5 apresenta as conclusões e lições aprendidas com essa experiência prática.

\section{Avaliações de Usabilidade e a Técnica WDP}

Os métodos comumente adotados para avaliação de usabilidade podem ser divididos em duas categorias: (1) Inspeções de Usabilidade, nas quais inspetores examinam aspectos da aplicação para detectar violações de princípios de usabilidade estabelecidos; e (2) Testes de Usabilidade, que são métodos de avaliação baseados na participação direta de usuários. Embora o teste de usabilidade seja considerado o método mais eficaz para avaliar sistemas e protótipos do ponto de vista do usuário das aplicações, seu custo é alto pois envolve o tempo dos usuários e muitas vezes o uso de laboratórios específicos de usabilidade (Matera et al., 2002). Os métodos de inspeção foram propostos como uma alternativa com bom custo-benefício em comparação com os testes de usabilidade.

$\mathrm{Na}$ pesquisa da técnica WDP (Web Design Perspectives-based Usability Evaluation), foi elaborada uma proposição na qual é testada se a adoção de perspectivas torna a Avaliação Heurística (Nielsen, 1994) mais eficiente para a inspeção de usabilidade de aplicações Web. Para isto foram utilizadas perspectivas próprias de projeto Web: Apresentação, Conceituação e Navegação (Conte et al., 2009a), empregadas como um guia para interpretar as heurísticas com foco específico em aplicações Web. A Figura 1 mostra um extrato da técnica WDP, para que se observe a diferença de enfoque na Heurística 9 ("Reconhecimento, diagnóstico e recuperação de erros") sob as perspectivas de Apresentação (par A.9), Conceituação (par C.9) e Navegação (par N.9). 
A.9. Reconhecimento, diagnóstico e recuperação de erros

- Avalie se as mensagens de erros estão claramente visíveis para o usuário

C.9. Reconhecimento, diagnóstico e recuperação de erros

- Avalie se as mensagens de erros utilizam linguagem simples de ser entendida pelos usuários, com conceitos do domínio do problema e de acordo com o perfil do usuário.

- Avalie se as mensagens de erros contém uma indicação de recuperação ou procedimento a ser executado que possa ser compreendida pelo usuário.

- Avalie se as soluções alternativas apresentadas nas mensagens de erro estão claras para o usuário.

N.9. Reconhecimento, diagnóstico e recuperação de erros

- Avalie se o sistema mostra como acessar as soluções alternativas apresentadas nas mensagens de erro.

\section{Figura 1 - Extrato da Técnica WDP}

A técnica WDP foi desenvolvida através de uma metodologia baseada em experimentação (Shull et al., 2001), que propõe uma seqüência de estudos experimentais com o objetivo de facilitar a sua transferência da academia para a indústria de forma segura. O presente artigo relata parte dos resultados obtidos com o estudo de caso de ambiente industrial. Um relato detalhado dos demais estudos realizados pode ser encontrado em (Vaz et al., 2008; Conte et al., 2009a; Conte et al., 2009b).

\section{Caracterização do Projeto e da Inspeção de Usabilidade}

\subsection{Objetivo e Indicadores da Avaliação}

O propósito da técnica WDP é poder ser empregada pelos próprios colaboradores das organizações de desenvolvimento, sem necessidade de contratação de especialista para a inspeção de usabilidade. O principal objetivo desta avaliação foi avaliar a aceitação e adequação da utilização da técnica em ambiente industrial. Isto foi medido através de dois indicadores: (a) Eficiência na etapa de Detecção, razão entre o número de defeitos por tempo de inspeção; (b) Opinião subjetiva do inspetor sobre a viabilidade de aplicação, já que os inspetores em questão são profissionais da indústria de software.

Segundo (Laitenberger e Dreyer, 1998) investigar a aceitação dos usuários para uma tecnologia requer um modelo que explique as atitudes e comportamentos das pessoas. Baseando-se no modelo de aceitação de tecnologia (Technology Acceptance Model - TAM) proposto por DAVIS (1989), esse indicador foi operacionalizado através de dois fatores: (1) Percepção sobre utilidade (Perceived usefulness), definida como "o grau no qual uma pessoa acredita que utilizar uma tecnologia específica melhoraria seu desempenho no trabalho" e (2) Percepção sobre facilidade de uso (Perceived ease of use), definida como "o grau no qual uma pessoa acredita que utilizar uma tecnologia específica seria livre de esforço" (Laitenberger e Dreyer, 1998). O modelo TAM tem sido aplicado amplamente a um grande conjunto de novas tecnologias (Venkatesh et al., 2003).

Dessa forma, neste estudo de caso, foi criado um questionário pós-inspeção com uma escala de 6 pontos, tendo como base os questionários aplicados por (Laitenberger e Dreyer, 1998) e (Denger et al., 2004) . Nesse questionário, os inspetores respondiam 
qual o seu grau de concordância em relação a afirmações sobre a utilidade ((1.a) A técnica WDP ajuda o inspetor a detectar defeitos; (1.b) A técnica WDP apóia a produtividade da inspeção (boa cobertura de defeitos em tempo razoável); (1.c) A técnica WDP torna a inspeção de usabilidade mais fácil para o inspetor) e sobre facilidade de uso ((2.a) A técnica WDP é de fácil utilização; (2.b) As perspectivas Web (Apresentação, Conceituação e Navegação) são fáceis de compreender; (2.c) Os itens dos pares HxP da técnica WDP são fáceis de compreender).

\subsection{Módulo iMPS do Ambiente de Apoio à Gerência de Operações do MPS.BR}

O objeto da inspeção foi o módulo i-MPS do sistema Ambiente de Apoio à Gerência de Operações do MPS.BR (Melhorias de Processo do Software Brasileiro) (Softex, 2009), aplicação Web gerada a partir do ambiente CORE-KM (Galotta et al., 2003). O módulo i-MPS foi desenvolvido para apoiar a execução do projeto de pesquisa sistemática para coleta de informações sobre resultados de desempenho de organizações que adotaram o modelo MPS (Kalinowski et al., 2008).

\subsection{Execução da Inspeção de Usabilidade}

O processo de inspeção utilizado teve por base o processo sugerido por SAUER et al. (2000). As atividades e os papéis que compõem o processo de inspeção de usabilidade utilizado são apresentados resumidamente na Tabela 1:

\section{Tabela 1- Atividades e papéis do Processo de Inspeção}

\begin{tabular}{|l|l|}
\hline Planejamento & $\begin{array}{l}\text { Definição do escopo da inspeção, preparação do material para a inspeção, seleção } \\
\text { dos inspetores e treinamento dos mesmos na técnica WDP. }\end{array}$ \\
\hline Detecção & $\begin{array}{l}\text { Cada inspetor executa individualmente essa atividade, a qual consiste na busca de } \\
\text { problemas de usabilidade nas interações selecionadas. }\end{array}$ \\
\hline Coleção & $\begin{array}{l}\text { Eliminação de discrepâncias repetidas (encontradas por mais de um inspetor), } \\
\text { gerando uma lista de discrepâncias únicas (sem duplicatas). }\end{array}$ \\
\hline Discriminação & $\begin{array}{l}\text { Classificação das discrepâncias em defeitos reais. As discrepâncias não classificadas } \\
\text { como defeitos são consideradas falso-positivos. }\end{array}$ \\
\hline Inspetores & $\begin{array}{l}\text { Responsáveis pela detecção das discrepâncias. Foram selecionados 5 inspetores: } 4 \\
\text { desenvolvedores da organização que não participaram da equipe de desenvolvimento } \\
\text { do i-MPS (com experiência em desenvolvimento entre 4 e 12 anos) e o 50 inspetor foi } \\
\text { um dos avaliadores de requisitos da aplicação, tendo experiência } \\
\text { desenvolvimento e inspeção de software (10 anos), porém sem atuação como } \\
\text { desenvolvedor na organização produtora de software. }\end{array}$ \\
\hline $\begin{array}{l}\text { Responsável } \\
\text { pelo Sistema }\end{array}$ & $\begin{array}{l}\text { Papel do gerente do projeto do módulo i-MPS. Não participou da detecção de } \\
\text { discrepâncias, porém atuou nas etapas de planejamento e discriminação. }\end{array}$ \\
\hline $\begin{array}{l}\text { Especialista na } \\
\text { Aplicação }\end{array}$ & $\begin{array}{l}\text { Papel do gerente sênior que colaborou com o gerente do módulo i-MPS. Devido ao } \\
\text { seu conhecimento da aplicação como um todo, ele colaborou com o Responsável } \\
\text { pelo Sistema na etapa de discriminação. }\end{array}$ \\
\hline Moderador & $\begin{array}{l}\text { Responsável pela execução do processo de inspeção como um todo e por atividades } \\
\text { específicas, como a coleção. Devido a restriçães de tempo dos colaboradores da } \\
\text { organização, esse papel foi exercido pela pesquisadora responsável pela técnica. }\end{array}$ \\
\hline
\end{tabular}

Planejamento - Os seis casos de uso do módulo i-MPS foram divididos em dois cenários: (1) cenário de administração de dados base para os questionários; (2) cenário de administração dos questionários, com as possibilidades de edição e exportação dos dados. Optou-se por criar roteiros com atividades que inspecionassem as funcionalidades dos dois cenários. Foram criados cinco roteiros, um para cada inspetor, com base na especificação do sistema e atividades equivalentes, apenas com variação nos dados para teste. O Responsável pelo Sistema fez a preparação da base de dados para a inspeção e a Moderadora, com auxílio de outros pesquisadores da técnica, inseriu os dados que 
seriam necessários às interações avaliadas. Também nesta etapa foi feita a capacitação dos inspetores na utilização da técnica WDP.

Detecção - Nesta atividade, os inspetores executaram as interações descritas no roteiro, e relataram as discrepâncias encontradas numa planilha, preenchendo as informações: Par Heurística x Perspectiva $(\mathrm{HxP})$ relacionado ao problema, identificação da atividade, descrição do problema, se o problema se repete em outras interações do sistema, e seu Grau de Severidade (seguindo a escala proposta por Nielsen (1994)).

Coleção - Após receber as planilhas com as discrepâncias enviadas por cada inspetor, a moderadora comparou as planilhas dos inspetores, verificando a existência de duplicatas. Foi criada uma lista única de discrepâncias para ser avaliada durante a reunião de discriminação. As duplicatas foram revisadas por dois outros pesquisadores da técnica.

Discriminação - Foi realizada uma única reunião de discriminação; estavam presentes a moderadora, o responsável pelo sistema e o especialista na aplicação. As interações avaliadas foram re-executadas, sendo assim possível verificar in-loco cada discrepância relatada. Após a apresentação da discrepância, o responsável pelo sistema e o especialista na aplicação a classificavam em defeito ou falso-positivo. Ao qualificar uma discrepância como defeito, o responsável pelo sistema revisava a classificação da severidade, considerando a ordem de prioridade a ser seguida para correção.

\section{Resultados Obtidos}

Um dos objetivos ao executar essa experiência foi observar se seria possível ter um bom aproveitamento da inspeção tendo como inspetores de usabilidade desenvolvedores da própria organização, dado que nenhum desses é um especialista em usabilidade. Nesta organização, todos os inspetores que realizaram a detecção já possuíam experiência anterior em execução de algum tipo de inspeção. No entanto nenhum deles possuía experiência prévia em execução de inspeções de usabilidade. A Tabela 2 mostra os tipos de experiência prévia de cada inspetor, o número de discrepâncias relatadas e quantas dessas discrepâncias foram classificadas como defeitos ou falso-positivos.

Tabela 2 - Caracterização dos Inspetores e Resultados da Inspeção do i-MPS

\begin{tabular}{|c|c|c|c|c|c|c|}
\hline $\begin{array}{l}\text { 홍 } \\
\stackrel{0}{0} \\
\stackrel{0}{0}\end{array}$ & $\begin{array}{l}\text { Experiência } \\
\text { inspeções }\end{array}$ & $\begin{array}{l}\text { Experiência } \\
\text { avaliações } \\
\text { usabilidade }\end{array}$ & $\begin{array}{l}\text { Experiência } \\
\text { Técnica WDP }\end{array}$ & $\begin{array}{l}\text { Número } \\
\text { Discrepâncias }\end{array}$ & $\begin{array}{l}\text { Número } \\
\text { Falso- } \\
\text { Positivos }\end{array}$ & $\begin{array}{l}\text { Número } \\
\text { Defeitos }\end{array}$ \\
\hline 1 & Sim & Não & Não & 11 & 1 & 10 \\
\hline 2 & Sim & Não & Não & 20 & 3 & 17 \\
\hline 3 & Sim & Não & Não & 16 & 6 & 10 \\
\hline 4 & Sim & Não & Não & 39 & 11 & 28 \\
\hline 5 & Sim & Não & Não & 11 & 3 & 8 \\
\hline
\end{tabular}

Em termos de tempo e esforço, o período dado aos inspetores para a execução da detecção de discrepâncias foi de uma semana. O esforço médio efetivamente gasto por cada inspetor foi de aproximadamente 2 horas e 38 minutos. Foram encontrados 73 defeitos no total e em média 14,6 defeitos por inspetor. O primeiro indicador, Eficiência na etapa de Detecção, foi calculado como a razão entre o número de defeitos por tempo de inspeção. $\mathrm{O}$ valor resultante foi de 5,55 defeitos/hora por inspetor, o que aponta um bom rendimento dos inspetores. 


\subsection{Análise segundo o Modelo de Aceitação de Tecnologia}

Em relação ao segundo indicador, Opinião subjetiva do inspetor sobre a viabilidade de aplicação da técnica, o questionário de percepção possuía uma escala de seis pontos: (1) Discordo Totalmente, (2) Discordo Amplamente, (3) Discordo Parcialmente, (4) Concordo Parcialmente, (5) Concordo Amplamente, (6) Concordo Totalmente. Não foi utilizada uma escala de sete pontos contendo um valor neutro intermediário pois, segundo Laitenberger e Dreyer (1998) este valor neutro não fornece informações sobre para qual direção o participante está inclinado (concordar ou discordar).

A Figura 2 mostra que todos os inspetores concordaram totalmente sobre a utilidade da técnica WDP na detecção de defeitos de usabilidade (grau relacionado à questão 1.a). Em relação ao grau de concordância sobre o aumento de produtividade da inspeção com a aplicação da técnica (questão 1.b), a maioria concordou, porém um dos inspetores discordou parcialmente. Essa resposta está de acordo com a sua produtividade na inspeção, pois este inspetor levou mais tempo que os outros na fase de detecção. Ainda sobre a percepção sobre a utilidade da técnica, verifica-se na Figura 3.a que todos os inspetores concordaram que a técnica WDP torna a inspeção mais fácil (questão 1.c) - dois inspetores concordaram totalmente, dois outros concordaram amplamente e um quinto inspetor concordou parcialmente.

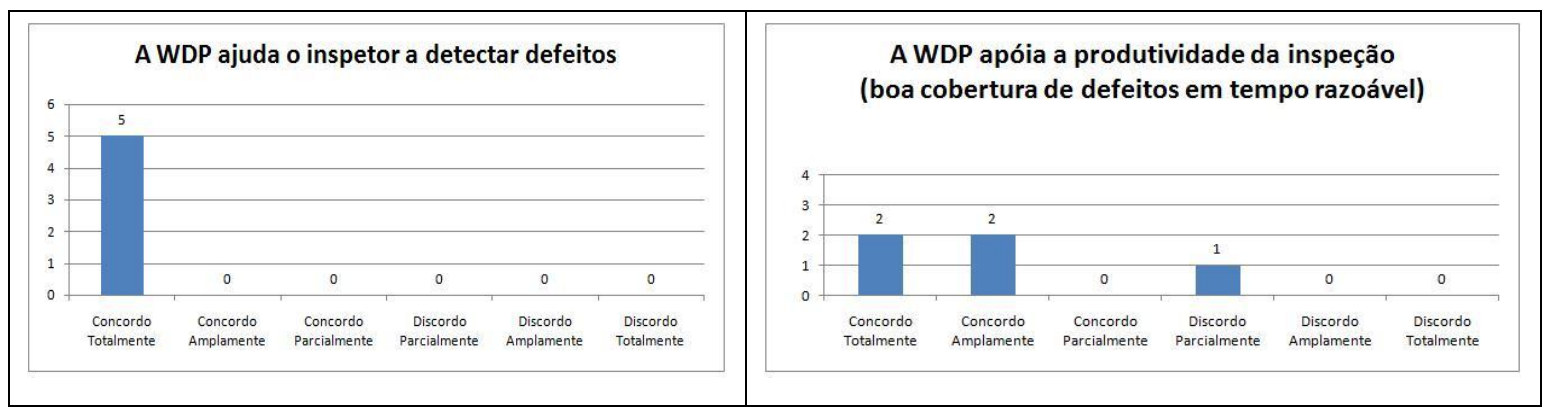

Figura 2 - Respostas sobre (a) Utilidade e (b) Produtividade da técnica WDP

Para as três afirmações relacionadas à percepção sobre facilidade de uso da técnica WDP não houve nenhuma resposta discordando. Em relação à facilidade de uso da técnica (questão 2.a - Figura 3.b), dois inspetores responderam concordando totalmente, um concordou amplamente e dois concordaram parcialmente. Sobre a facilidade de compreensão tanto das perspectivas Web (questão 2.b) quanto dos pares HxP (questão 2.c), como pode ser visto na Figura 4, três inspetores concordaram amplamente, um concordou totalmente e um concordou parcialmente.

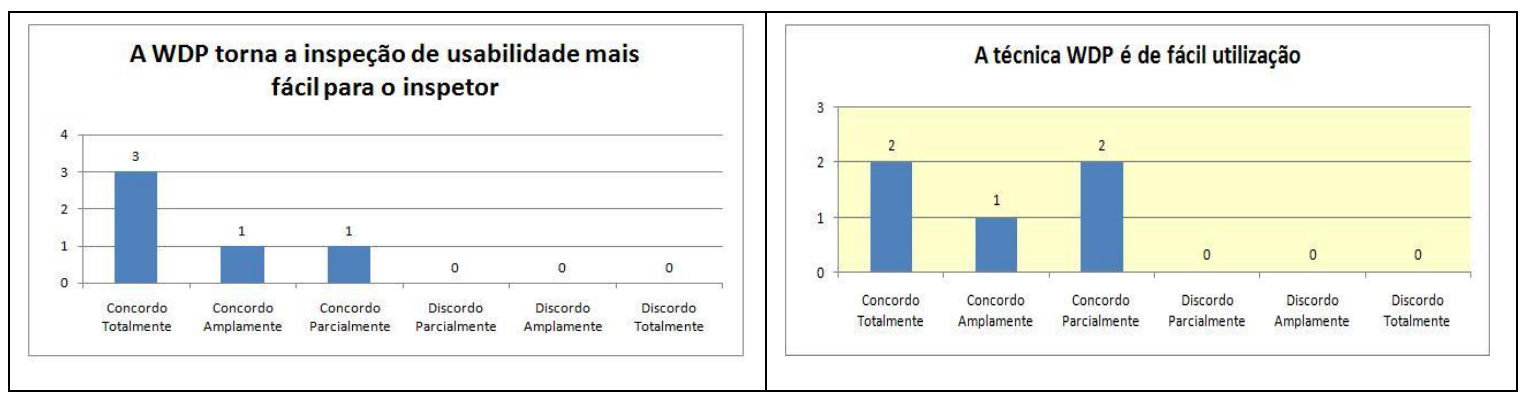

Figura 3 - Respostas (a) se a técnica WDP torna a inspeção mais fácil e (b) sobre a Facilidade de uso da técnica WDP 


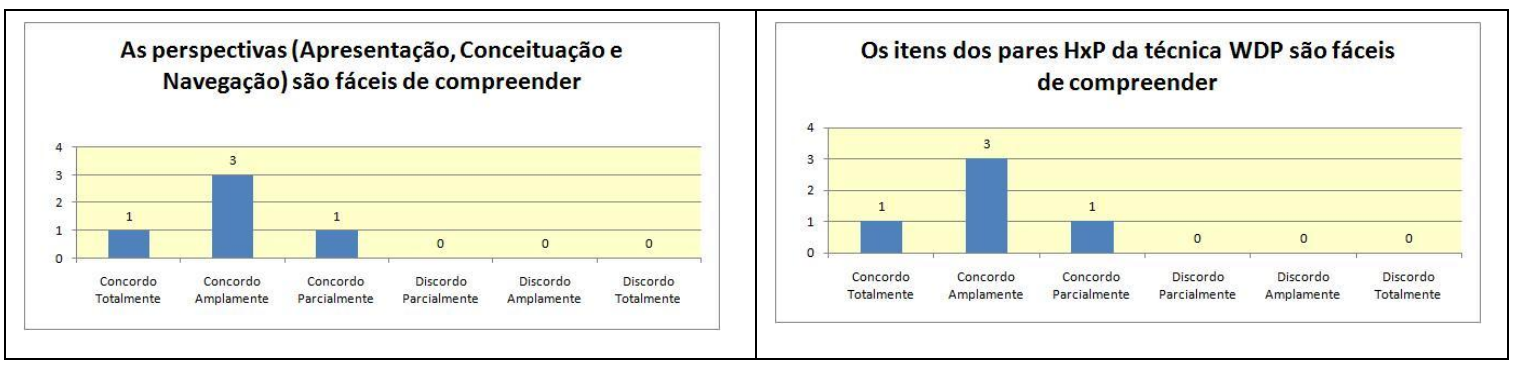

Figura 4 - Respostas (a) sobre Facilidade de Compreensão das Perspectivas e (b) sobre Facilidade de Compreensão dos Pares HxP

Uma limitação desse estudo de caso foi o número de participantes, que não permite o teste estatístico dos dados. Embora os resultados desse estudo de caso não possam ser generalizados, os resultados quantitativo e qualitativo apóiam a indicação obtida em estudo anterior (Vaz et al., 2008) sobre a viabilidade de utilização dos próprios desenvolvedores e avaliadores de requisitos como inspetores em uma avaliação de usabilidade, utilizando-se a técnica WDP.

\section{Considerações Finais}

Este trabalho apresentou o relato da execução de uma inspeção de usabilidade em um projeto de desenvolvimento de software. Em adição aos resultados discutidos em (Vaz et $a l ., 2008)$, pode-se observar que é viável realizar uma inspeção de usabilidade com os próprios desenvolvedores de um projeto de software. Nessa experiência o custo da inspeção se mostrou baixo, pois o esforço médio de um inspetor na atividade de detecção foi de 2 horas e 38 minutos e na detecção foram encontrados 73 defeitos (com média de 14,6 defeitos por inspetor). A relação de eficiência da inspeção foi de 5,5 defeitos/hora de inspeção por inspetor. Espera-se com estes resultados incentivar a indústria de software Web a realizar avaliações de usabilidade com maior freqüência.

É importante ressaltar que, apesar de ser interessante comparar este resultado em termos de eficiência com outras técnicas de avaliação de usabilidade, tal comparação não é trivial. A dificuldade está no fato de que não faz sentido que um inspetor re-inspecione um mesmo caso de uso. Desta forma, em um estudo comparativo, seria necessário mudar o inspetor e/ou o objeto da inspeção, dificultando uma comparação direta dos resultados.

A utilização do modelo TAM para avaliação da técnica WDP visa encorajar o uso deste modelo na avaliação de aceitação de novas tecnologias de software.

\section{Agradecimentos}

Os autores agradecem a todos os profissionais que participaram da inspeção e aos pesquisadores Emilia Mendes, Jobson Massolar e Ulysses Vilela por todas as suas contribuições durante o desenvolvimento da técnica WDP. Agradecemos à SOFTEX, ao CNPq e à FAPEAM pelo apoio.

\section{Referências}

Conte, T., Massolar, J., Mendes, E., et al., 2009a, "Web Usability Inspection Technique Based on Design Perspectives", IET Software Journal, v. 3, n. 2 (April 2009), pp. 106-123.

Conte, T., Vaz, V., Massolar, J., et al., 2009b, "Improving a Web Usability Inspection Technique using Qualitative and Quantitative Data from an Observational Study". XXIII Simpósio Brasileiro de Engenharia de Software, pp. 227 - 235. 
Davis, F., 1989, "Perceived usefulness, perceived ease of use, and user acceptance of information technology", MIS Quarterly, v. 13, n. 3, pp. 319-339.

Denger, C., Ciolkowski, M., Lanubile, F., 2004, "Investigating the Active Guidance Factor in Reading Techniques for Defect Detection". Proc. of the 2004 International Symposium on Empirical Software Engineering (ISESE'04), v. 1.

Galotta, C., Zanetti, D., Rocha, A.R., et al., 2003, "CORE-KM: Um Ambiente Customizável para Gerência de Conhecimento", $S B C$, Fortaleza.

Hitz, M., Leitner, G., Melcher, R., 2006, "Usability of Web Applications". In: KAPPEL, G., PRÖLL, B., REICH, S., et al. (eds), Web Engineering: The Discipline of Systematic Development of Web Applications, John Wiley \& Sons.

Insfran, E., Fernandez, A., 2008, "A Systematic Review of Usability Evaluation in Web Development ". Proc. of 2nd Int. Workshop on Web Usability and Accessibility (IWWUA 2008), v. LNCS 5176 - WISE 2008 Workshops, pp. 81-91, Auckland.

Kalinowski, M., Weber, K.C., Travassos, G.H., 2008, "iMPS: an experimentation based investigation of a nationwide software development reference model". Proc. of the 2nd ACM-IEEE Int. Symposium on Empirical software engineering and measurement, pp. 336-338, Kaiserslautern, Germany, October.

Laitenberger, O., Dreyer, H.M., 1998, Evaluating the Usefulness and the Ease of Use of a Web-based Inspection Data Collection Tool, IESE.

Matera, M., Costabile, M.F., Garzotto, F., et al., 2002, "SUE Inspection: An Effective Method for Systematic Usability Evaluation of Hypermedia", IEEE Transactions on Systems, Man and Cybernetics, Part A, v. 32, n. 1 (Jan 2002), pp. 93-103.

Nielsen, J., 1994, "Heuristic evaluation". In: JAKOB NIELSEN, MACK, R.L. (eds), Usability inspection methods, New York, NY, John Wiley \& Sons, Inc.

Olsina, L., Covella, G., Rossi, G., 2006, "Web Quality". In: MENDES, E., MOSLEY, N. (eds), Web Engineering, Spinger Verlag.

Sauer, C., Jeffery, D.R., Land, L., et al., 2000, "The Effectiveness of Software Development Technical Reviews: A Behaviorally Motivated Program of Research", IEEE Transactions on Software Engineering, v. 26, n. 1, pp. 1-14.

Shull, F., Carver, J., Travassos, G.H., 2001, "An empirical methodology for introducing software processes", ACM SIGSOFT Software Engineering Notes, v. 26, n. 5, pp. 288-296.

Softex, 2009, "Site do MPS.BR - Melhoria de Processos do Software Brasileiro". In: http://www.softex.br/mpsBr, acessado em Janeiro/2010.

Travassos, G.H., 2008, "Experimentação em Engenharia de Software: produzindo evidências, desenvolvendo tecnologias e melhorando processos de software (Apresentação Keynote)" SBQS 2008.

Travassos, G.H., Barros, M., 2003, "Contributions of In Virtuo and In Silico Experiments for the Future of Empirical Studies in Software Engineering". Proc. of the 2nd Workshop in Workshop Series on Empirical Software Engineering (WSESE 2003), pp. 117-130.

Vaz, V., Conte, T., Bott, A., et al., 2008, "Inspeção de Usabilidade em Organizações de Desenvolvimento de Software - Uma Experiência Prática". Anais do VII Simpósio Brasileiro de Qualidade de Software (SBQS 2008), v. 1, pp. 369-378.

Venkatesh, V., Morris, M.G., Davis, G.B., et al., 2003, "User acceptance of information technology: Toward a unified view", MIS Quarterly, v. 27, n. 3, pp. 425-478. 